\title{
STRUCTURAL CHANGES IN DEPTH SECTION AS A RESULT OF VELOCITY MODEL INCORRECTNESS
}

\author{
DOI: http://dx.doi.org/10.18509/GBP.2016.26
}

UDC: 550.344.094.5:[621.386.1:004.6

\author{
Assist. Prof. Dr. Maya Grigorova \\ University of Mining and Geology “St. Ivan Rilski”, Bulgaria
}

\begin{abstract}
Building proper velocity model for Prestack depth migration can bring significant improvement of seismic data quality (both resolution and continuity) and proper spatial structural positioning. For these reasons special attention is required on creating accurate initial velocity model in time domain, as a starting point for building reliable updated depth velocity field for Prestack depth migration.
\end{abstract}

Keywords: Depth migration, velocity field, tomography, structural changes

\section{INTRODUCTION}

Normal moveout, typically used to do velocity analysis in seismic data processing, is robust when reflectors are flat and velocity is laterally invariant. However, this method encounters difficulties when reflectors are dipping or velocity varies laterally. In areas of rapid lateral and vertical velocity changes, energy is dispersed in such a way that conventional stacking velocities cannot provide desired resolution of the data. A good initial model limits ambiguity and increases convergence of tomographic solutions. Therefore, an initial model plays key role in a process of migration velocity analysis for complex structures. In the case of tomographic inversion, a wrong provisional velocity distribution makes it impossible to obtain convergent results [1].

\section{PRESTACK DEPTH MIGRATION WITH INITIAL VELOCITY MODEL}

Nowadays, the requirements for obtaining high resolution seismic sections lead to searching different approaches to process the observed data. Creating depth sections by Pre-Stack Depth Migration (PSDM) is a key issue for obtaining more reliable and informative seismic sections, which in turn enable more confident and more reliable interpretation of seismic data.

Depth imaging of seismic data is used to map the complex subsurface geology in areas that have steeply dipping structures, faults or in any areas with a rapidly changing geological environment. The main aspect for achieving high quality depth imaging is the ability to build detailed and accurate velocity model. The model building process involves making an initial estimate of interval velocities, followed by iterative refinement of the velocity field by residual moveout which has been minimized on depth migrated gathers. Additionally, the velocity model created for PSDM carries direct knowledge about geology. Still, our goal is to obtain a section which is as close as possible to a geological cross-section. For these reasons, velocity estimation process is the main task in depth imaging. Accurate velocity estimation and depth imaging provides the key to understanding the subsurface. 
On figure 1 is shown PSDM workflow:

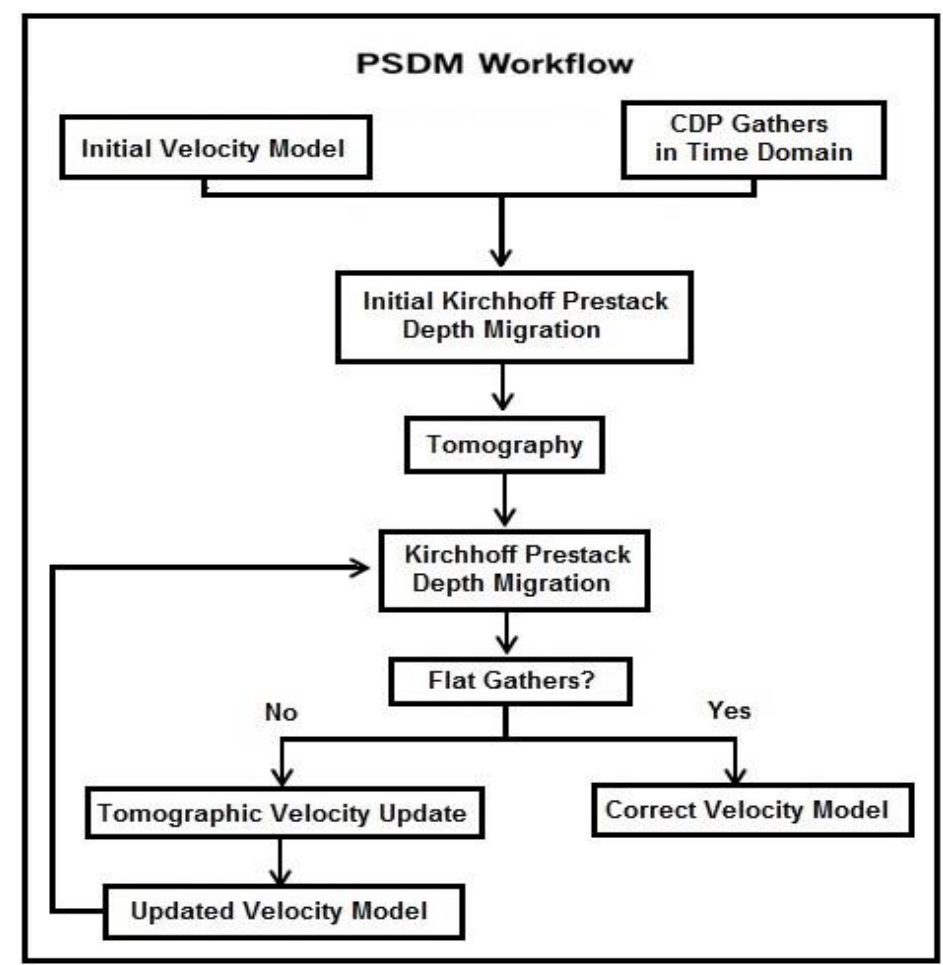

Figure 1. Prestack Kirchhoff Depth Migration (PSDM) workflow

The input data for initial prestack depth migration are processed CDP gathers in time domain and initial RMS interval velocity model. For experimental purposes two velocity models are defined and with every one of them is performed PSDM:

- Velocity model with no rapid changes in velocity trend - the original;

- Velocity model with rapid changes in velocity trend - artificially created.

The derived velocity fields are interpolated and smoothed using proprietary software. This ensures that both the seismic data and velocity fields tie following the migration process [2].

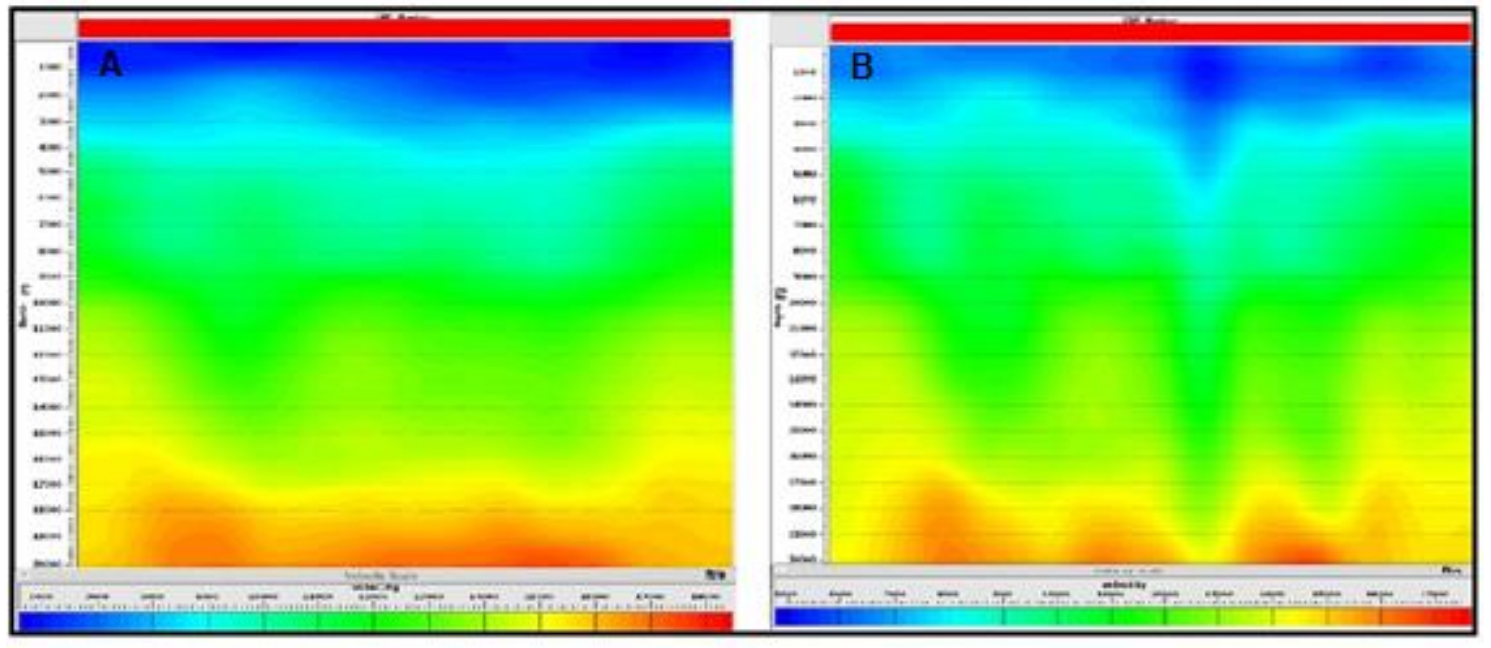

Figure 2. Initial velocity models: A - velocity model with no rapid changes in velocity trend; B - velocity model with rapid changes in velocity trend 
In arias of complex geology sometimes it is difficult to follow general velocity trends and it resulting as inhomogeneous and complicated velocity field with rapid changes in velocity trend. The effect that it has on PSDM is shown on figure 3.

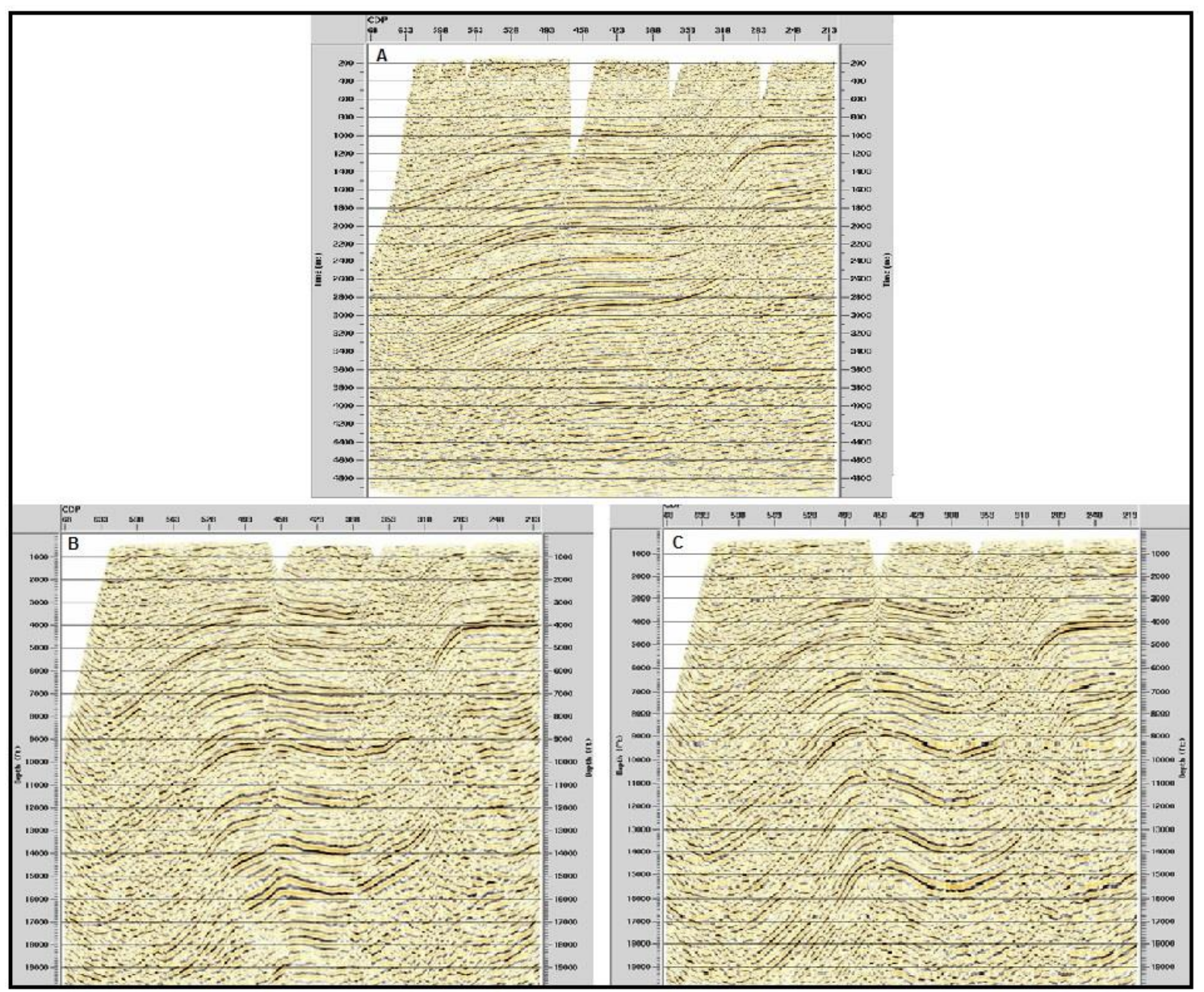

Figure 3. A - Prestack Time Migration; B - Preliminary PSDM with velocity model with no rapid changes in velocity trend; C - Preliminary PSDM with velocity model with rapid changes in velocity trend

On figure $3 \mathrm{~A}$ is shown the result of Prestack Time Migration performed with the initial velocity model. On the picture in time domain the slope of the reflection boundaries is small in the middle of the image and it does not suppose that in depth domain could be much different. Nevertheless these velocity fields are used for tomography inversion process on the next stage of the work.

\section{TOMOGRAPHIC INVERSION}

The quality of initial velocity distribution has the greatest influence on tomographic inversion process. Even small velocity variations can bring to significant structural changes on the seismic section after PSDM within only several iterations of tomography. For these reasons special attention is focus on this step.

Seismic tomography compares observed travel times, measured for each source-receiver pair, with expected travel times, computed by ray tracing through an assumed velocity 
model [3]. This information is used as input to sophisticated tomography solution for direct inversion into an updated interval velocity model.

In the first phase of the method the initial model is updated by tomographic inversions and lateral velocity changes are defined.

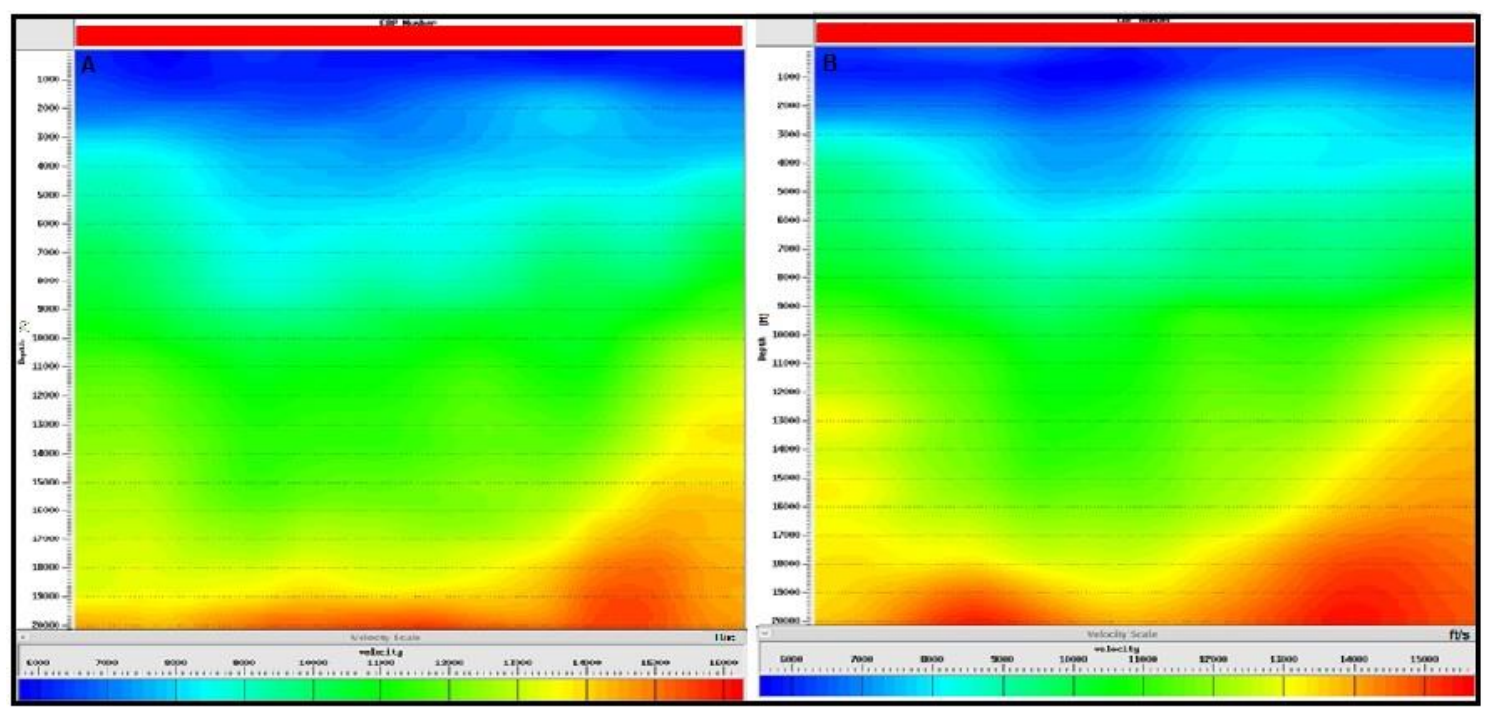

Figure 4. A - velocity model with no rapid changes in velocity trend after tomography; B - velocity model with rapid changes in velocity trend after tomography

Successive iterations are performed until the obtained structural model could be accepted and tomographic inversion velocities updates reached limits of $\pm 250 \mathrm{~m} / \mathrm{s}$ [4]. The number of iterations was data-dependent but usually 2-4 iterations were sufficient [1]. After building velocity models corresponding to that limits PSDM is performed.

\section{PRESTACK DEPTH MIGRATION USING TOMOGRAPH BASED VELOCITY MODEL}

The resulting depth images with velocity models performed by tomography inversion are shown with on figure 5 .

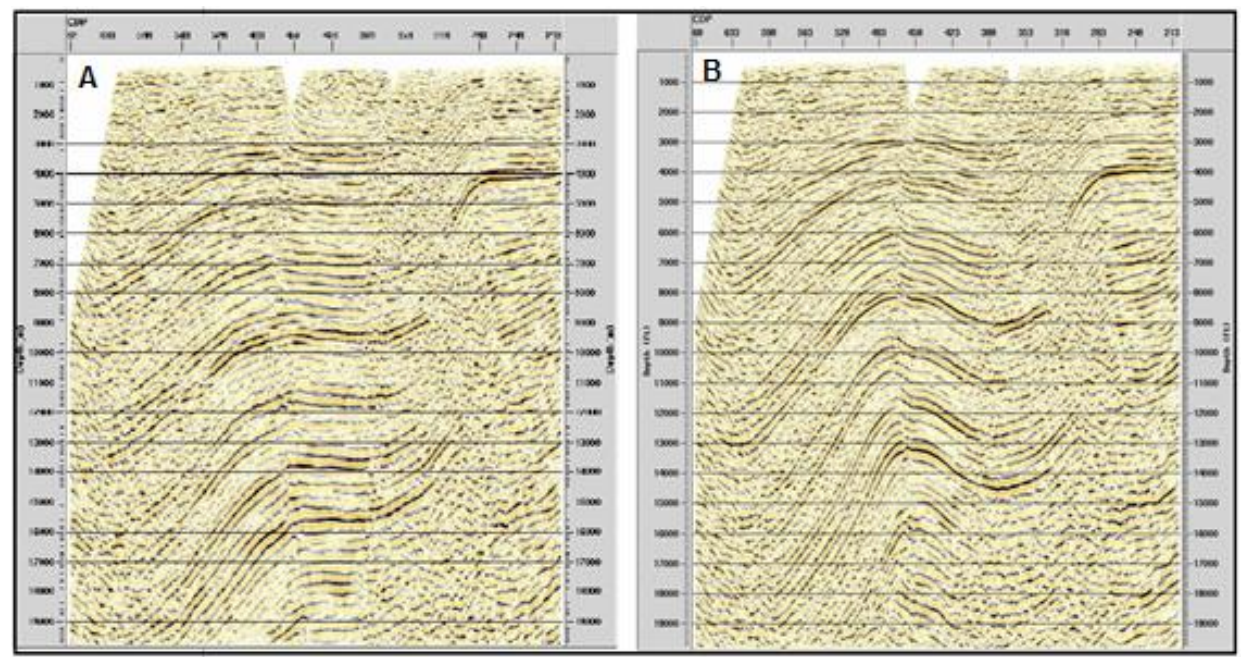

Figure 5. Final stack data after PSDM with: A - velocity model with no rapid changes in velocity trend after tomography; B - velocity model with rapid changes in velocity trend after tomography 
Both depth migrations show improved structural features with better standouts of the events than the first passes of the PSDM with the initial velocity models.

Comparing the two sections after PSDM with tomography based velocity fields, it is obvious that the velocity model with rapid changes in velocity trend bring to artificial slopes in the depth migrated section. Structural changes are observed in this model due to lack of homogeneity in the initial velocity field.

Furthermore better definition and continuity of horizons were received on the PSDM section with this final iteration of tomography inversion for the model without rapid changes in velocity trend. This led to more reliable structural definition of the model.

\section{CONCLUSION}

Successful depth imaging requires careful attention on velocity model building process. There is no doubt that PSDM provides informative and reliable results when it is performed with accurate velocity model. Building velocity model for depth imaging is tend to be great challenge. The main reason is the fact that the solution is non-unique and both experience and dynamic model building procedures are required for fast, efficient and accurate velocity model estimation. This study shows that variations in starting velocity field can cause structural distortions of depth migrated images and make them inadequate for accurate geological interpretation of subsurface structures.

\section{REFERENCE}

[1] Grigorova, M., Dabovski, D., How many iterations are necessary for obtaining proper depth velocity model?, 8th Congress of Balkan Geophysical Society, Greece, 2015, No

[2] Grigorova, M. Time - to - Depth migration using tomography based velocity model, 7th Congress of Balkan Geophysical Society, Albania, 2013, No 18499.

[3] Johannes A. van Trier [1990], Tomographic determination of structural velocities from depth-migrated seismic data, Stanford Exploration Project No 66, 5-6.

[4] Sliż, K., K., Prestack depth migration velocity model building in complex Carpathians geology, 53, № 3, pp. 311-324, 2005. 